\title{
Approximation Algorithms for the Firefighter Problem: Cuts over Time and Submodularity
}

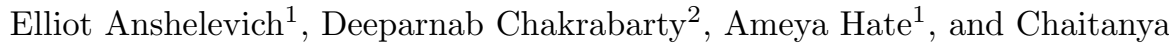 \\ Swamy $^{2}$ \\ 1 Department of Computer Science, Rensselaer Polytechnic Institute \\ ${ }^{2}$ Dept. of Combinatorics \& Optimization, University of Waterloo
}

\begin{abstract}
We provide approximation algorithms for several variants of the Firefighter problem on general graphs. The Firefighter problem models the case where an infection or another diffusive process (such as an idea, a computer virus, or a fire) is spreading through a network, and our goal is to stop this infection by using targeted vaccinations. Specifically, we are allowed to vaccinate at most $B$ nodes per time-step (for some budget $B$ ), with the goal of minimizing the effect of the infection. The difficulty of this problem comes from its temporal component, since we must choose nodes to vaccinate at every time-step while the infection is spreading through the network, leading to notions of "cuts over time".

We consider two versions of the Firefighter problem: a "non-spreading" model, where vaccinating a node means only that this node cannot be infected; and a "spreading" model where the vaccination itself is an infectious process, such as in the case where the infection is a harmful idea, and the vaccine to it is another infectious idea. We give complexity and approximation results for problems on both models.
\end{abstract}

\section{Introduction}

Faced with an epidemic that is spreading through a population, and a limited supply of vaccine (or simply a lack of time to administer it), it is necessary to decide whom to vaccinate. Questions about the spread of disease and epidemics in a social network have often been modeled using graph theory (e.g. [3,11]), and correspond to fundamental graph-theoretic concepts [22]. Moreover, these graph theoretic principles can be applied to many diffusive network processes, including epidemics in computer networks, the spread of innovations and ideas, and viral marketing [23]. In this paper, we focus specifically on inhibiting the spread of an epidemic or an idea by using vaccination.

Model and the Firefighter problem We model our network of agents as a directed ${ }^{3}$ graph $G=(V, E)$ and a source node $s$. All nodes in the graph can have one

\footnotetext{
${ }^{3}$ We use a directed graph since it is more general - an undirected graph is just a directed graph with two arcs per edge.
} 
of three states: they can be infected, vaccinated, or vulnerable, that is neither vaccinated nor infected. At time $\tau=0$, all nodes are vulnerable, except node $s$, which is infected. At each $\tau>0$, any vulnerable vertex $v$ which is connected to an infected node $u$, such that $(u, v) \in E$, gets infected at time $\tau+1$, unless it is vaccinated at time step $\tau$. Infected and vaccinated nodes stay infected and vaccinated respectively. We call a node saved if it is either vaccinated or if all paths from any infected node to it contains at least one vaccinated node.

Definition 1. A vaccination strategy is a set $\Psi \subseteq V \times J$ where $V$ is the set of vertices of graph $G$ and $J=\{1,2, \ldots|V|\}$. The vertex $v$ is vaccinated at time $\tau \in J$ by the vaccination strategy $\Psi$ if $(v, \tau) \in \Psi$. A vaccination strategy $\Psi$ is valid with respect to budget $B$, if the following two conditions are satisfied:

i. if $(v, \tau) \in \Psi$ then $v$ is not infected at time $\tau$,

ii. let $\Psi_{\tau}=\{(v, \tau) \in \Psi\}$; then $\left|\Psi_{\tau}\right| \leq B \quad$ for $\quad \tau=1 \ldots|V|$.

The first condition implies we can only vaccinate vulnerable nodes, and the second condition requires that no more than $B$ nodes are vaccinated at any time-step.

We consider two separate objectives in this paper. The first objective, which we call MAXSAVE, is to maximize the number of non-infected nodes in the end, when we are given a fixed budget $B$. The second objective, which we call MinBudget, is to minimize the budget $B$ needed per time instant in order to save a given set of nodes, $T \subseteq V$. They can be formally described as follows.

$\operatorname{MaxSave}(G, B, s, T)$

InstAnCE: A rooted graph $(G(V, E), s)$, integer $B \geq 1$ and $T \subseteq V$

OвJECTIVE: Find a valid vaccination strategy $\Psi$ such that if $s$ is the only infected node at time 0 , then at the end of the above process the number of non-infected nodes that belong to $T$ is maximized.

This problem is also referred to as the Firefighter PROBLEM in the literature when $T=V[16,20]$.

$\operatorname{MinBudget}(G, s, T)$

InstAnCE: A rooted graph $(G(V, E), s)$, and $T \subseteq V$

OBJECTIVE: Find a valid vaccination strategy $\Psi$ with minimum possible budget $B$, such that if $s$ is the only infected node at time 0 , then at the end of the above process all nodes in $T$ are saved.

We also consider a variant of this model in the paper, where the vaccination is also a process that spreads through the network. In this Spreading Vaccination Model, the vaccination spreads to all its neighboring nodes which are still vulnerable, thereby vaccinating them. That is, at time step $\tau>0$, if a node $v$ is vaccinated and there is a vulnerable node $u$ such that $(v, u) \in E$, then at time $\tau+1$, the node $v$ also gets vaccinated. Thus, the vaccination also spreads like the infection. Note that a vulnerable node can be adjacent to both an infected node and a vaccinated node. We will assume that the vaccine prevails over the infection, and in the subsequent time step, the vulnerable node is vaccinated, 
rather than being infected. This is actually a weak assumption as assuming otherwise doesn't change the quality of our results. In the spreading model, we will say that a node is vaccinated directly when it is vaccinated by the vaccination strategy, and it is vaccinated indirectly when it is vaccinated by the spread of the vaccine through the network.

Example 1. To gain some intuition about this problem, consider the example shown in Figure 1 using the non-spreading model of vaccination.

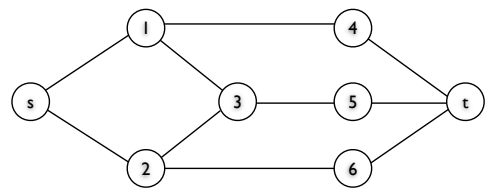

Fig. 1. This example shows that sometimes vaccinating nodes far away from the infection is the only way to save all the required nodes.

Consider the MinBudget objective for this example. The infection begins at node $s$, and the goal is to find the smallest number $B$ of nodes that need to be vaccinated at every time step so that we can save the node $t$, which we assume cannot itself be vaccinated. If we were only allowed to cut nodes during the first time-step, this would be equivalent to the minimum $s$ - $t$ node-cut problem. However, unlike previous works, such as [22], which examine the static problem of vaccinating a 'cut' before the infection has started spreading, we need to find the "best" cut over time (where best depends on the considered objective). This temporal nature of the problem, complicates matters: intuitively, the tradeoff is between vaccinating a small set of nodes close to the infection source early, or spreading out (over time) the vaccination of a larger set of nodes which are farther away from the source.

For instance, in the above example, a minimum $s$ - $t$ node-cut is $\{1,2\}$, which requires $B=2$. However, there $i s$ a solution to the above problem with $B=1$, but the final set of vaccinated nodes does not form a minimum $s$ - $t$ node-cut. One such solution is to vaccinate vertices 4,6 , and 5 at time steps 1,2 , and 3 respectively, leading to the final set of vaccinated nodes being $\{4,5,6\}$ which is not a minimum cut. In fact, it is not hard to come up with examples where the optimal value of $B$ is much smaller than the size of a minimum node $s$ - $t$ cut and the final set of vaccinated nodes is much larger than the size of a minimum node $s$ - $t$ cut (e.g., take a graph where $s$ has $k$ neighbours, each of which is connected to $t$ via $k$ long internally node-disjoint paths). Thus, this "cuts over time" problem is quite different from the classical min-cut problem, and in fact is known to be NP-hard (even when the graph is a tree!) [15]

Our Results In Section 2, we consider the model of spreading vaccinations. In general, our results show that this model is more tractable than the model with 
non-spreading vaccinations. For MAXSAVE we show that this problem reduces to maximizing a submodular function with a matroid constraint. Therefore a simple greedy algorithm provides a 2-approximation, and a recent result of [6] lets us prove a $(1-1 / e)$ factor approximation. For MinBudget we give a $O(\log n)$ approximation algorithm, and show that this approximation ratio is tight, by showing a set-cover hardness.

The non-spreading model, on the other hand, does not yield itself to good approximation algorithms. In fact, we show in Section 3 that it is NP-hard to approximate MAXSAVE in general graphs by a factor of $n^{\alpha}$, for any $\alpha<1$. For MinBudget, we give a $O(\sqrt{n})$ factor approximation algorithm for general graphs based on a natural LP relaxation for the problem. We also show that the integrality gap of the LP is bounded by $\Omega(\log n)$. For directed layered graphs with $\ell$ layers, we give a $O\left(H_{\ell}\right)=O(\log \ell)$ approximation algorithm. The latter algorithm is combinatorial and requires just one max-flow computation.

Section 3.1 is devoted to vaccination strategies when the underlying graph is a tree. This special case has received a lot of attention $[21,26]$, is computationally difficult [15], and is in fact a generalization of a complex scheduling problem (details in the full version [1]). For this special case we show that both the spreading and the non-spreading models are equivalent, so the stronger results from Section 2 hold for the non-spreading model as well. In addition, our algorithm for layered graphs also implies a $O(\log h)$ approximation algorithm for MinBudget on trees with height $h$. Note that this is stronger than the $O(\log n)$ algorithm we have for general graphs in the spreading model.

Related Work Questions about epidemic propagation have been studied in several fields, (e.g., [4,29]), although most of this research models the epidemics as dynamic systems and ignores the effect of the network structure. Recently, a few groups have considered the spread of viruses or ideas on Internet-like topologies, such as small-world networks [32] and preferential attachment models [5, 25]. Several papers also study targeted vaccinations in this context $[10,13]$, and show that they can be used to significantly reduce the effect of epidemics. These studies assume certain properties of the networks (based on where these networks arise from).

Several recent papers considered modeling vaccination by using graph cuts. For example, the work of Hayrapetyan et al. [22] and others [3,12] fully utilizes the social-network structure to "cut off" and contain various diffusive processes in a social network. As mentioned earlier, all this work is only concerned with vaccinating a set of nodes before the infection begins, however, and does not have the temporal component of the Firefighter problem. A lot more work has been done on maximizing the spread of an infection (instead of trying to stop it using vaccinations), by selecting the best nodes to infect initially $[11,23]$.

The Firefighter problem was first introduced by B. Hartnell [20], and there has been much work on this problem; see, e.g., [16] for a survey. However, much of the work has focused on special graph structures, such as grids [9,18,31], and that too usually with the MAXSAVE objective. The Firefighter problem is NPcomplete even when the underlying graph is a tree [15], although [21] and [26] 
give approximation algorithms for this case, and [28] shows how to solve the problem in in polynomial time for special cases of trees.

Independent of our work, Chuzhoy and Chalermsook recently obtained some similar results [7] for the non-spreading version of the the MinBudget problem. In particular, they give an $O(\log h)$-approximation for an $h$-layered directed graph (which matches our approximation ratio), and obtain a better approximation for trees.

\section{Spreading Vaccination Model}

We first show a few simple hardness results about this model, and then give approximation algorithms for both our objectives. Due to lack of space, all our proofs appear in the full version [1] of the paper.

\subsection{General Properties}

We make certain useful observations about this model. Let $N(v, i)$ be the set of all the nodes that are a distance of at most $i$ from $v$.

Lemma 1. At time $\tau$, all nodes in the neighborhood $N(s, \tau)$ will either be vaccinated or infected.

Now, since all the nodes in the neighborhood $N(s, \tau)$ will be either infected or vaccinated by time $\tau$, any optimal vaccination strategy would not vaccinate any node in this neighborhood at time $>\tau$. Since any valid strategy can vaccinate only $B$ nodes at any time-step, it means that an optimal strategy would vaccinate at most $B \cdot \tau$ nodes directly in the neighborhood $N(s, \tau)$.

We define a set $\Gamma(v)$ for every node $v \in V$ by

$$
\Gamma(v)=\{(u, \tau) \mid u \in V \text { and } 0<\tau \leq(d(s, v)-d(u, v))\}
$$

The tuple $(v, \tau)$ essentially represents the event of vaccinating node $v$ at time $\tau$. Using these definitions we state the following theorem.

Theorem 1. A node $v \in V$ is vaccinated by the vaccination strategy $\Psi$ iff $\Psi \cap \Gamma(v) \neq \emptyset$.

This theorem tells us that vaccinating an element of $\Gamma(v)$ is exactly what is needed in order to save a node $v$, and this gives us insight into the structure of the problem.

\subsection{Approximation for MaxSave}

As we show in the full version of the paper, the MAXSAVE problem can be modeled as a problem of maximizing a submodular set function on a collection of sets that form a partition matroid. On the basis of this knowledge, techniques like the greedy algorithm [17] can be used to obtain a $\frac{1}{2}$ approximation for MAXSAVE, while the randomized algorithm of [6] can be used to obtain a $(1-1 / e)$ approximation. 
Theorem 2. There is a randomized algorithm which gives with high probability $a(1-1 / e)$ approximation for the MAXSAVE problem. Additionally, a simple greedy algorithm gives a $\frac{1}{2}$ approximation.

The detailed proof is presented in the full version; here, we give its gist. A partition matroid consists of disjoint sets $E_{1}, \ldots, E_{k}$, and a set $S$ is called independent if $S \cap E_{i} \leq \ell_{i}$, for some given numbers $\ell_{1}, \ldots, \ell_{k}$. We argue that one can actually consider vaccination strategies that satisfy only property (ii) in Definition 1. This set of strategies forms a partition matroid, since we can only choose at most $B$ nodes at every time-step to vaccinate (so $\ell_{i}=B$ for all $i$ ). We next show that the function $f(\Psi)$ defined (suitably) as the number of nodes saved by using the (possibly invalid) vaccination strategy $\Psi$ is submodular, by using Theorem 1, and if $\Psi$ satisfies the budget-constraint then there is a valid vaccination strategy $\Psi^{\prime}$ such that $f(\Psi)=f\left(\Psi^{\prime}\right)$. We then use the results of $[6,17]$ to obtain the desired approximations.

It should be noted here that the same $(1-1 / e)$ approximation can also be obtained by applying a randomized rounding technique similar to [26] to a modified version of the MAXSAVE problem. We believe, however, that modeling the problem using partition matroids and submodular functions gives an algorithm that is combinatorial in nature, deterministic and hence more efficient.

\subsection{Approximation for MinBudget}

Theorem 3. The MinBudget problem is $\log n$ inapproximable by reduction from SET Cover.

Consider an instance of MinBudget. First suppose that we know the size of the optimal budget $B$ that is needed in order to save all nodes of $T$. Below we give an algorithm that saves all nodes in $T$ using a budget of at most $B \log n$. To form a $\log n$ approximation algorithm without knowing $B$, we simply do a binary search on $B$, and run the algorithm below every time.

By slight adjustments to the proof of Theorem 2, we know that by running the greedy algorithm with budget $B$, we save at least half of the nodes in $T$. The greedy algorithm in this case chooses the nodes to vaccinate in each time-step one at a time, always picking the node that saves the most nodes of $T$. For this purpose the greedy algorithm needs to know exactly which nodes will be saved if a node $u$ is vaccinated at time $\tau$, which we can compute in poly-time. Once finished with the first time-step, the algorithm goes on to the second, and so on. The full algorithm for MinBudget is as follows.

Repeat $\log (n)$ times:

- vaccinate nodes in graph $G$ using the greedy algorithm with budget $B$.

- Construct graph $G_{1}$ from $G$ by removing all the vertices that were vaccinated directly and indirectly in the previous step. Let $T_{1}$ be the nodes of $T$ that are in $G_{1}$.

- Set $G=G_{1}$ and $T=T_{1}$. 
It is clear that the new graph $G_{1}$ will always contain the original source node $s$ as it is never vaccinated by the greedy algorithm. By repeating the application of the greedy algorithm that vaccinates $B$ nodes at every time-step $\log n$ times, we end up with an algorithm that vaccinates $B \log n$ nodes at every step. Let this be the RepGreedy algorithm.

Theorem 4. The algorithm RepGreedy saves all nodes of $T$ by vaccinating at most $B \log n$ per time-step.

For the final $\log n$ approximation algorithm to MINBUDGET, do binary search on $B$, and run RepGreedy for every choice of $B$.

\section{Non-Spreading Vaccination Model}

The non-spreading model is considerably more difficult than the spreading model. One of the main reasons is that Lemma 1 (or any simple modification of it) is no longer true.

The MaxSave problem is NP-complete for bipartite graphs [28] and for cubic graphs (3-regular) [24]. The MAXSAVE problem is NP-complete even when restricted to trees with maximum degree three [15]. We prove the following about the inapproximability of MAXSAVE

Theorem 5. The MaxSave $(G(V, E), s, B, T)$ problem cannot be approximated in poly-time to the factor of $n^{\alpha}$ where $n=|V|$ and $\alpha<1$, unless $P=N P$.

We introduce an auxiliary problem, SAVE-t, which asks whether a specified node $t$ can be saved by vaccinating one node (other than $t$ ) at a time. The NPcompleteness of this problem follows from known NP-completeness proofs. We then give a gap introducing reduction from the SAVE-t problem to the MAXSAVE problem such that if there exists any $n^{\alpha}$ approximation for the MAXSAVE problem then we can solve the SAVE-t problem in polynomial time. The proof appears in full version.

In the remainder of the section, we focus on the MinBudget problem. Note that we need to save all the nodes in a set $T$ with the minimum number of vaccinations required per time instant. To simplify notation, we consider the following equivalent problem: we add a new node $t$ with edges from all nodes in $T$ to $t$, and consider the problem of saving $t$ with minimum budget under the additional constraint that $t$ itself cannot be vaccinated. We call $s$ the source and $t$ the sink. Let $\mathcal{P}$ denote the collection of all $s$ - $t$ paths. We start with the following LP relaxation of the problem and its dual.

The primal LP has a variable $x_{v}^{\tau}$ which indicates whether vertex $v$ is vaccinated at time $\tau$ or not. $\ell \leq n$ is the length of the longest path from $s$ to $t$; it is easy to see that we will not vaccinate any vertex after time $\ell$. The first constraint bounds the number of vaccinations at every time instance. The second constraint says that for every path $\left(s, v_{1}, \cdots, v_{k}, t\right)$ to the sink $t$, one of the nodes, say $v_{i}$, must be vaccinated by time $i$. This is a necessary and sufficient condition for this 


$$
\begin{aligned}
& \begin{array}{l}
\text { Minimize } \\
\qquad \sum_{v \in V} x_{v}^{\tau} \leq B \quad \forall \tau=1, \ldots, \ell
\end{array} \\
& \text { (Primal) } \quad \text { Maximize } \quad \sum_{P \in \mathcal{P}} f_{P} \\
& \sum_{\tau=1}^{\ell} z_{\tau} \leq 1 \\
& \sum_{i=1}^{k} \sum_{\tau=1}^{i} x_{v_{i}}^{\tau} \geq 1 \quad \forall\left(s, v_{1}, \cdots, v_{k}, t\right) \in \mathcal{P} \\
& x_{v}^{\tau} \geq 0, \quad \forall v \in V, \forall \tau=1, \ldots, \ell \\
& z, f \geq 0
\end{aligned}
$$

path not to transmit the infection to $t$. In the dual, we have a flow for every $s$ - $t$ path $P$. We also have a variable $z_{\tau}$ which add up to 1 . The second constraint in the dual is a bit subtle: it says, for every $\tau$, the total flow through a vertex $v$ via paths such that $v$ lies at a distance $\tau$ or more from $s$ on the path, is at most $z_{\tau}$. In the LP, $P^{(\tau)}$ denotes the portion of the path from the $\tau$ th vertex to $t$. That is if $P=\left(s, v_{1}, \ldots, v_{k}, t\right)$, then $P^{(\tau)}=\left(v_{\tau}, v_{\tau+1}, \ldots, t\right)$.

Although the primal LP above has exponentially many constraints, it can be solved in polynomial time since one can obtain the separation oracle in polynomial time. Strictly speaking the LP (Primal) may have an integrality gap of $n=|V|$. However note that if $O P T$ denotes the optimal value of (Primal), then in fact $\lceil O P T\rceil$ is a lower bound on the minimum budget, and by comparing the budget of our solution against this lower bound, we prove the following theorems. We only give proof sketches deferring the full proofs to the full version.

Theorem 6. In the non-spreading model, there is a $2 \sqrt{n}$ approximation to the MinBudGeT problem in general graphs.

(Proof Sketch) At a high level, the algorithm recognizes the set of vertices to be vaccinated by time $i$ by looking at the fraction vaccinated by time $i$. If this fraction is larger than $1 / \sqrt{n}$, then the node is vaccinated by day $i$. We can then show that in the remaining graph, infection can reach $t$ only using paths of length longer than $\sqrt{n}$, and thus there is a cut of size $\sqrt{n}$ which separates $s$ and $t$. Thus vaccinating this cut as well completes the algorithm. The analysis is slightly subtle and is deferred to the full version.

An $s$ - $t$ directed layered graph with $\ell$ layers is one where (i) $s$ has only outgoing edges, $t$ has only incoming edges; (ii) all nodes except $t$ can be partitioned into sets $L_{0}:=\{s\}, L_{1}, L_{2}, \ldots, L_{\ell}$ such that for every node $v \in L_{i+1}$ (so $v \neq t$ ) and every incoming edge $(u, v)$ of $v$, we have $u \in L_{i}$.

Theorem 7. If the network is a layered directed graph with $\ell$ layers, then there is a $H_{\ell}$ approximation to the MinBudget problem. Furthermore, there is an example which shows that the integrality gap of an layered network is at least $H_{\ell}=\Omega(\log n)$, where $H_{r}=1+1 / 2+\cdots+1 / r$.

(Proof Sketch) The algorithm sets capacity $1 / i H_{\ell}$ on each vertex of layer $i$, for all $i$, and simply computes a minimum $s$ - $t$ vertex cut. It then divides the cut 
into $\ell$ pieces, corresponding to the vertices vaccinated on day $i$. Using the dual of the LP, we can show that our solution is within $H_{\ell}$ of the LP optimum. The integrality gap example is a similar layered graph. We defer the details to the full version.

\subsection{Vaccination on Trees}

When $G$ is a tree rooted at $s$, the following observation establishes the equivalence between the spreading model and non-spreading model. For the spreading model on general graphs we defined a function $\Gamma(v)$ as a set of all tuples $(u, \tau)$ such that if $u$ is vaccinated directly at time $\tau$ then the node $v$ will be saved. For a tree, it is easy to observe that a node $v$ will be saved if any of its ancestors is vaccinated directly before the infection reaches $v$. Therefore, the optimal strategy will be the same on a given tree irrespective of the vaccination model being spreading or non-spreading. This implies that all the positive results from Section 2 also hold for trees. Since the MinBudget problem on trees with height $h$ yields an instance of MinBudget on an $s$ - $t$ directed graph with $h$ layers, we immediately obtain the following Corollary of Theorem 7 .

Corollary 1. There is a $O(\log h)$ approximation for MinBudget on trees, where the set $T$ is the set of leaves.

\section{Acknowledgments}

The authors would like to thank David Kempe, Sanjeev Khanna, Jon Kleinberg and, Malik Magdon-Ismail for interesting and productive discussions on the Firefighter problem.

\section{References}

1. Full version can be found at www.cs.rpi.edu/ eanshel/pubs.html

2. R. Ahuja, T. Magnanti, and J. Orlin. Network Flows: Theory, Algorithms, and Applications. Prentice Hall, 1993.

3. J. Aspnes, K. Chang, and A. Yamposlkiy. Inoculation strategies for victims of viruses and the sum-of-squares partition problem. In Proc. 16th ACM SODA, 2005.

4. N. Bailey. The Mathematical Theory of Infectious Diseases and its Applications. Hafner Press, 1975.

5. A. Barabasi, R. Albert, and H. Jeong. Mean-field theory for scale-free random networks. Physica A, 272, 1999.

6. G. Calinescu, C. Chekuri, M. Pal, J. Vondrak. Maximizing a Monotone Submodular Function subject to a Matroid Constraint. 12th International IPCO Conference, Ithaca, NY, USA, 2007.

7. P. Chalermsook, J. Chuzhoy. Resource Minimization for Fire Containment. to appear in Proc. ACM SODA, 2010.

8. Stuart Crosby, Art Finbow, Bert Hartnell, Raina Moussi, Kate Patterson and Dania Wattar. Designing Fire Resistant Graphs. Congr. Numerantium 173, 2005. 
9. M. Develin, S.G. Hartke. Fire Containment in grids of dimension three or higher. Discrete Applied Math,155(17), 2007.

10. Z. Dezső and A. Barabási. Halting viruses in scale-free networks. Physical Review E, 65, 2002.

11. P.A. Dreyer Jr. and F. S. Roberts. Irreversible k-threshold processes: Graphtheoretical threshold models of the spread of disease and of opinion. Discrete Applied Mathematics, 157(7), 2009.

12. R. Engelberg, J. Könemann, S. Leonardi, and J. Naor. Cut problems in graphs with a budget constraint. In Proc. 7th LATIN, 2006.

13. S. Eubank, V. Kumar, M. Marathe, A. Srinivasan, and N. Wang. Structural and algorithmic aspects of massive social networks. In Proc. 15th ACM SODA, 2004.

14. U. Feige A threshold of $\ln n$ for approximating set cover, J. ACM 45, 1998.

15. S. Finbow, A. D. King, Gary MacGillivray, R. Rizzi. The Fire fighter problem on graphs of maximum degree three. Discrete Mathematics 307, 2007.

16. Stephen Finbow, Gary MacGillivray The Firefighter Problem: A survey of results, directions and questions. manuscript, 2007.

17. M.L. Fisher, G.L. Nemhauser and L.A. Wolsey An analysis of approximations for maximizing submodular set functions - II. Math. Prog. Study, 8:7387, 1978.

18. P. Fogarty. Catching Fire on Grids, M.Sc. Thesis, Department of Mathematics, University of Vermont 2003.

19. G. Giakkoupis, A. Gionis, E. Terzi, P. Tsaparas. Models and algorithms for network immunization. Technical Report C-2005-75, Department of Computer Science, University of Helsinki, 2005.

20. B.L. Hartnell. Firefighter! An application of domination. Presentation, 25th Manitoba Conference on Combinatorial Mathematics and Computing, University of Manitoba in Winnipeg, Canada, 1995.

21. B. Hartnell, Q. Li. Firefighting on trees: How bad is the greedy algorithm? Congr. Numer. 145, 2000.

22. A. Hayrapetyan, D. Kempe, M. Pal and Z. Svitkina Unbalanced graph cuts. In ESA, Mallorca, Spain 2005.

23. D. Kempe, J. Kleinberg and E Tardos. Influential Nodes in a Diffusion Model for Social Networks. In Proceedings of the 32nd ICALP, 2005.

24. Andrew King, Gary MacGillivray. The Firefighter Problem For Cubic Graphs. Discrete Mathematics, 307, 2007.

25. R. Kumar, P. Raghavan, S. Rajagopalan, D. Sivakumar, A. Tomkins, and E. Upfal. Stochastic models for the web graph. In Proc. 41st IEEE FOCS, 2000.

26. C. Leizhen, E. Verbin and L. Yang Firefighting on Trees: (1 - 1/ e )Approximation, Fixed Parameter Tractability and a Subexponential Algorithm, ISAAC 2008, 2008.

27. Cai Leizhen and Wang Weifan. The Surviving Rate of a Graph. to appear in SIAM Journal on Discrete Mathematics, 2009.

28. Gary MacGillivray, P. Wang. On The Firefighter Problem JCMCC, 47, 2003.

29. M. Nowak and R. May. Virus Dynamics: Mathematical Principles of Immunology and Virology. Oxford University Press, 2000.

30. R. Raz and S. Safra. A sub-constant error-probability low-degree test, and subconstant error-probability PCP characterization of NP. In Proc. 29th ACM STOC, 1997

31. P. Wang, S. Moeller Fire Control on graphs. J. Combin. Math. Combin. Comput., 41,2002 .

32. D. Watts and S. Strogatz. Collective dynamics of 'small-world' networks. Nature, 393, 1998. 